\title{
O PERFIL E AVALIAÇÃO DA ASSISTÊNCIA TÉCNICA A PRODUTORES DE LEITE NA MICROREGIÃO DE SÃO JOÃO BATISTA DO GLÓRIA/MG
}

\author{
Ricardo Ferreira Godinho ${ }^{1}$ \\ Rita de Cássia Ribeiro Carvalho \\ Vando Edesio Soares ${ }^{3}$
}

\section{Resumo}

Inúmeras mudanças e transformações vêm alterando o cenário agrícola e agrário brasileiro despertando para a necessidade de repensar a demanda e oferta de profissionais que atendam às unidades produtivas do agronegócio, quanto às reais necessidades das empresas e ao perfil dos profissionais que as assistem. $O$ objetivo deste trabalho foi identificar e descrever o perfil dos profissionais de ciências agrárias que atendem os produtores de leite do município de São João Batista do Glória/MG. Os dados foram levantados diretamente dos produtores rurais e profissionais da assistência técnica atuantes no município. Uma restrição à maior participação dos técnicos no processo administrativo das empresas agropecuárias diz respeito ao sigilo de informações, aliado ao vínculo comercial que a maioria dos técnicos possui, e uma relativa insegurança dos produtores rurais em expressar sua opinião sobre o assunto. O baixo uso de técnicas de gestão nos sistemas de

\footnotetext{
1 Graduado em Zootecnia (UNESP), Mestre em Produção Animal (UNICASTELO), Especialista em Administração Rural (UFLA), Consultoria Organizacional (SEBRAE). Professor Graduação e Pós Graduação (UEMG Passos).E-mail: ricardo.godinho@fespmg.edu.br

${ }_{2}^{2}$ Zootecnista (UFLA), Mestre e Doutora em Zootecnia (Forragicultura e Pastagem UFLA). Professor adjunto da FESP-Universidade do Estado de Minas Gerais e orientadora de Iniciação Científica.E-mail: rita.carvalho@fespmg.edu.br

${ }^{3}$ Zootecnista (UNESP), Mestre e Doutor (UNESP). Professor Coordenador do Curso de Mestrado Profissional em Produção Animal UNICASTELO. E-mail: soaresvando@gmail.com
} 
produção leiteira possui uma relação com o baixo envolvimento dos profissionais da assistência técnica e com as questões administrativas dessas empresas, as quais exploram estes sistemas, embora existam diferentes opiniões e percepções sobre o problema, tanto por parte dos produtores quanto dos profissionais da assistência técnica.

Palavras-chave: Ciências agrárias, Extensão rural, Gestão do agronegócio, Perfil profissional, Qualificação profissional.

\title{
TECHNICAL ASSISTANCE TO MILK PRODUCTION SYSTEMS IN THE MICRO-REGION OF SÃO JOÃO BATISTA DO GLÓRIA/MG
}

\begin{abstract}
Several changes and transformations have been altering the Brazilian agrarian and agricultural setting bringing the need to rethink the offer and demand of professionals who meet the agribusiness productive units when it comes to the companies' real necessities and the professionals' profiles who assist them. The objective of this paper was to identify and describe the Science Agrarian professionals' profiles of those who assist the milk producers in the municipality of São João Batista do Glória/MG. The data were surveyed directly from the rural producers and technical assistance professionals who work in the municipality. A restraint regarding a bigger participation of technicians in the administrative process of agricultural companies is about information secrecy linked to the commercial bond that most of the technicians have, and certain insecurity from rural producers to express their opinions about the topic. The low use of management techniques in milk production systems has a relation with the low involvement of the technical assistance professionals with administrative issues, although there are different opinions and perceptions about the problem, coming from the producers and technical assistance professionals.
\end{abstract}

Key-words: Agribusiness management, agricultural sciences, Extension, professional profile, professional qualification. 


\section{INTRODUÇÃO}

Inúmeras mudanças e transformações vêm alterando o cenário agrícola e agrário brasileiro, de país essencialmente agrícola, - Brasil transformou-se num país urbano. Entre estas transformações, destacam-se as inovações tecnológicas e estruturais observadas nas últimas décadas no setor agrícola brasileiro, decorrentes de avanços significativos da pesquisa; as rupturas nas tradições, nas formas de expressão e nas relações humanas, tanto no campo quanto nas áreas urbanas; a diminuição das distâncias de espaço e tempo; a forte demanda da sociedade por produtos agrícolas que sejam, ao mesmo tempo, ambientalmente limpos, seguros para o consumo e de baixo preço; a crescente responsabilidade social do setor agrícola para atingir produção recorde ano a ano, fixação de mão-de-obra no campo, aumento da oferta de alimentos e o superávit da balança comercial (CCAUFSCAR, s/d).

Mesmo diante deste cenário o setor rural brasileiro tem passado por dificuldades há muito não enfrentadas, em que se conjugam problemas climáticos, sanitários, macroeconômicos e de mercado para a maioria dos produtos do agronegócio.

Para Batalha et al. (2005a), contrapondo a estas dificuldades, há alguns anos, o agronegócio tem se mostrado um dos setores mais importantes da economia brasileira, não só pela renda gerada, mas também pela geração de empregos; entretanto, os empregos gerados requerem profissionais qualificados e com novas habilidades, uma vez que as empresas de agronegócio atuam em um mercado global e competitivo. Este cenário desperta para a necessidade de repensar a demanda e oferta de profissionais que atendam às unidades produtivas do agronegócio, quanto às reais necessidades das empresas e ao perfil dos profissionais que as assistem.

Batalha et al. (2005b), em estudo sobre a formação e disponibilidade de recursos humanos para o agronegócio aponta que as empresas buscam no profissional um perfil mais generalista, em que as habilidades pessoais e de comunicação adquirem grande importância em relação às habilidades técnicas e específicas. Além disso, verificou uma enorme preocupação das empresas com relação aos conhecimentos e habilidades dos profissionais em economia e gestão, que possibilitam maior visão sistêmica do agronegócio, 
fazendo com que possam trabalhar analiticamente a partir de uma visão de todo o sistema agroindustrial, e não apenas de macrossistemas isolados. Acredita-se que as empresas esperam que os profissionais utilizem-se das ferramentas gerenciais de maneira a compreender a dinâmica de funcionamento das cadeias agroindustriais, de maneira a torná-las mais eficientes e eficazes.

A necessidade de formar profissionais com um perfil sintonizado com as demandas sociais do campo vem sendo apontada como um dos principais desafios a serem enfrentados pelas universidades brasileiras, neste princípio de século. Tal desafio, no entanto, defronta-se com uma série de obstáculos, oriundos em grande parte da orientação excessivamente tecnicista e produtivista dos conteúdos programáticos presentes nas grades curriculares dos cursos das ciências agrárias, desde meados da década de 1960, quando o ensino agrícola, a pesquisa agropecuária e a extensão rural foram estruturadas de modo a atender aos interesses da modernização da agricultura, também conhecida por 'revolução verde' (ROS, 2008).

Com o objetivo de identificar as transformações no agronegócio brasileiro, demonstrando o perfil dos profissionais demandados pelas empresas que atuam no sistema agroindustrial brasileiro e confrontá-lo com a formação de ensino superior que está sendo oferecida nesta área no Brasil, Batalha et al. (2005a) concluíram, entre outros aspectos, que o maior desajuste ocorreu em relação aos conteúdos de Economia e Gestão, principalmente ao avaliar os cursos que exploram as áreas mais técnicas do agronegócio; em seguida, foram apontados os desajustes com relação às habilidades e conhecimentos do grupo de Qualidades Pessoais, Métodos Quantitativos e Sistemas de Informação, além de um preocupante desajuste em relação às habilidades de comunicação. Tais desajustes segundo estes autores devem ser avaliados com bastante critério, uma vez que as empresas buscam por um perfil profissional mais generalista, em que as habilidades pessoais e de comunicação sobressaem-se em relação às habilidades técnicas e específicas.

Neste mesmo trabalho, Batalha et al (2005a), ao hierarquizar a pontuação média realizada pelo conjunto de todas as empresas atuantes no agronegócio (Qualidades Pessoais: 8,88 pontos; Comunicação e Expressão: 7,69 Pontos; Economia e Gestão: 7,13 Pontos; Métodos Quantitativos Computacionais e 
Sistemas de Informação: 6,15 Pontos; Tecnologia de Produção: 5,99 Pontos; e Experiência Profissional Desejada: 4,88 Pontos), deixaram claro que nos dias atuais, as empresas esperam de um profissional mais do que as habilidades técnicas adquiridas durante o curso superior, esperam que seus funcionários sejam pró-ativos e participem intensamente do cotidiano da empresa, não só na solução de problemas, mas também na visualização de novas oportunidades de negócio; Para tanto, são muito importantes os conhecimentos e as habilidades pessoais, como flexibilidade, iniciativa, capacidade para a tomada de decisão, negociação, trabalho em grupo e alto padrão ético, e os relativos à capacidade de expressão e de interação/relacionamento com outros profissionais. Um aspecto que reforça tais resultados é que eles foram confrontados com pesquisa semelhante realizada em 1998, e pouco foi alterada, havendo apenas uma inversão entre "Métodos Quantitativos Computacionais e Sistemas de Informação" e "Tecnologia de Produção".

Conforme afirmou Batalha et al. (2005b) o mercado exige um novo perfil para o profissional do agronegócio. No entanto, os profissionais que já atuam no campo necessitam rever suas atitudes, e segundo pesquisa de Canziani (2001), há por parte dos profissionais esta abertura para a capacitação em temas relativos à gestão do agronegócio.

Um levantamento das principais tendências do mundo acadêmico relacionadas à Extensão Rural no conjunto das universidades públicas e privadas do Brasil, foi realizado por Callou et al. (2008); estes autores concluíram que há uma insuficiência da carga horária necessária à formação do extensionista/gestor de processos de desenvolvimento local, mesmo considerando a contribuição dos conteúdos programáticos de outras disciplinas correlatas à Extensão Rural; esta questão tende a se agravar quando se constata que a disciplina Extensão Rural é, quase sempre, oferecida nos últimos semestres dos Cursos de Ciências Agrárias, podendo dificultar uma formação continuada do aluno no âmbito das discussões que se voltam para os contextos rurais, corroborando ainda para o fato de que este aspecto seria um elemento que obscurece o caráter multidisciplinar da Extensão, impedindo-a ainda de desempenhar a função de elo condutor das demais disciplinas do curso.

Há uma necessidade imediata de reformulação na estrutura de profissional em ciências agrárias, no sentido de um sólido 
conhecimento teórico-prático não apenas ao "como produzir", mas também quanto ao processo produtivo como um todo, abrangendo desde o planejamento até a comercialização. Além disso, torna-se fundamental o domínio da informática com todas suas nuances e da língua inglesa, pelo menos. Portanto, o profissional formado deve ter uma visão interdisciplinar de todo o processo para que se torne um profissional valorizado (MAIA et al., 2006).

Realizando uma reflexão crítica sobre o ensino superior no Brasil na área de agronegócios, Rinaldi et al. (2007) destacaram que a importância do agronegócio para a economia nacional e as mudanças que ele vem atravessando, nos âmbitos nacional e internacional, tem alimentado uma demanda por profissionais diferenciados dos habitualmente formados pelos cursos tradicionais da área de Ciências Agrárias.

Para Batalha et al. (2005a) é importante refletir se os cursos de ensino superior estão formando profissionais que consigam compreender o agronegócio de maneira integrada e se as disciplinas estão sendo abordadas realmente de maneira multidisciplinar, de forma que os mesmos consigam entender a crescente complexidade do segmento agroindustrial, que acresceu dificuldades no gerenciamento dos agronegócios e portanto, exige ferramentas gerenciais mais sofisticadas.

O Brasil somente passará a ter profissionais competitivos para atuarem em sistemas agroindustriais, a partir do momento em que a formação de professores e pesquisadores priorizar o perfil multidisciplinar e sistêmico presente na lógica da organização das cadeias produtivas. Este é um problema em cadeia, que precisa urgentemente ser estudado, pois a cada ano novos cursos de pósgraduação e, principalmente de graduação, são criados, e são eles que definirão o perfil de profissionais que irão estabelecer o padrão de competitividade do Agronegócio brasileiro (RINALDI et al, 2007).

Em relação à sazonalidade da produção de leite no país, Gomes (2006) aponta que existem duas forças antagônicas atuando: a primeira a favor, predominante nos sistemas de produção a pasto, enquanto a segunda força contra, é impulsionada pela modernização da pecuária e pela indústria, em razão da ociosidade causada pelo sistema sazonal. A tendência é reduzir a sazonalidade, sem contudo, eliminá-la.

A visão holística do agronegócio e a interdependência entre os atores que compõem o sistema agroindustrial do leite será um 
fator decisivo na relação e resultados obtidos pelos técnicos e produtores. Segundo Zylbersztajn (2003), hoje se pensa em uma relação interdependente, com conflito e cooperação convivendo e sendo gerenciados; a pauta de ações que decorre da mudança de foco da produção para o consumo e de uma percepção estática para uma relação dinâmica entre os agentes atuantes no Sistema Agroindustrial (SAG) é totalmente distinta para todos os envolvidos. A assistência técnica e extensão rural, que antes levavam as informações aos produtores, estão sendo revistas para atuarem como facilitadoras da comunicação entre produtores e as fontes de informação (SONKA, 1997 apud ZYLBERSZTAJN, 2003).

Acompanhando e analisando a estrutura gerencial de uma propriedade familiar localizada no Passo do Pilão, em Pelotas - RS, Reichert (1998) enfocou a administração e o gerenciamento durante um ano agrícola e avaliou os índices técnicos e econômicos das atividades desenvolvidas. Neste trabalho, 0 autor concluiu que 0 processo de acompanhamento técnico, contábil e gerencial permitiu que o produtor em foco tomasse conhecimento, por meio de contatos mensais, dos resultados parciais ou totais de cada atividade, podendo organizar e planejar melhor o ano agrícola seguinte ou as próximas tarefas. Dessa forma, pôde tomar as decisões com base em fatos concretos e com conhecimento de causa.

De acordo com o Diagnóstico da Pecuária Leiteira em Minas Gerais (GOMES, 2006), metade dos produtores entrevistados não foi visitada em sua propriedade para receber orientação sobre gado de leite, o que confirmou a pequena abrangência da assistência técnica individual. Dos entrevistados, $26 \%$ receberam uma ou duas visitas, número inexpressivo para uma orientação capaz de causar mudanças no processo produtivo. Quanto aos meios de comunicação de massa, $73 \%$ dos entrevistados assistem ao programa de televisão Globo Rural e programas de rádio, que no passado eram muito citados, têm frequência de apenas $7,8 \%$.

Por este mesmo Diagnóstico, $11,50 \%$ dos entrevistados responderam que têm carência de informações sobre planejamento da empresa rural; $16,15 \%$, sobre cálculo do custo de produção; e $23 \%$, sobre mercado de leite, que dizem respeito ao gerenciamento da empresa rural. Na opinião dos entrevistados de todas as regiões do Estado, eles não têm carência de informações sobre produção de leite e meio ambiente. Segundo o autor, eles ainda não estão sensibilizados para a importância desses temas. 
Confrontando os resultados dos diagnósticos realizados em Minas Gerais nos anos de 1995 e 2005, constatou-se diferenças na opinião dos entrevistados sobre carência de treinamento. Em 1995, pouca importância foi dada ao tema gerenciamento da empresa rural, enquanto em 2005 este foi a principal citação. Quanto ao meio ambiente, os produtores ainda não perceberam a importância deste tema.

Um aspecto preocupante apontado no Diagnóstico de 2005 (GOMES, 2006) foi quanto ao resultado proporcionado pelas ações de capacitação. Questionados sobre os efeitos das informações que receberam nos treinamentos, $22 \%$ dos produtores responderam que estas não contribuíram para mudanças na produção de leite; $22 \%$, que não contribuíram para melhorar a qualidade da mão-de-obra; e $20 \%$, que não contribuíram para aumentar a produtividade do rebanho. Segundo o autor, essas respostas contradizem as dos produtores sobre a qualidade das informações, já que a maioria respondeu que estas são de boa qualidade. Tal contradição recomenda avaliações mais aprofundadas sobre o conteúdo dos treinamentos e, mais ainda, sobre os efeitos destes, pois qual seria a importância de realizar muitas palestras e dias-de-campo, se tais realizações, na opinião dos produtores, não contribuem com mudanças na produção do leite.

No que diz respeito à assistência técnica em Minas Gerais, segundo este mesmo diagnóstico, de modo geral, os produtores entrevistados não utilizam frequentemente os serviços oferecidos pela indústria laticinista, tanto das cooperativas quanto da indústria particular; na maioria das vezes, esse serviço nem é oferecido. Verificou-se tendência de extinção dos departamentos de assistência técnica, em razão do elevado custo e da pouca efetividade destes. Diversas indústrias preferem terceirizar a assistência técnica, mediante programas como o Educampo e outros da mesma natureza. Entre os serviços profissionais mais utilizados, o dos veterinários é citado por $12,64 \%$ dos entrevistados; o dos agrônomos, por 5,37\%; e, finalmente, o dos zootecnistas, por apenas $1,52 \%$. A frequência maior dos veterinários reflete o tipo de demanda por assistência técnica curativa. Sem uma visão preventiva, na assistência técnica os efeitos são, geralmente, pouco significativos, razão da explicação para o fechamento de muitos departamentos de assistência técnica. 
Esta pesquisa foi realizada com o objetivo de descrever o perfil dos profissionais de ciências agrárias que atendem os produtores de leite do município de São João Batista do Glória/MG, e avaliar o trabalho da assistência técnica, segundo a percepção dos produtores.

\section{MÉTODOS}

Os dados para esta pesquisa foram levantados diretamente dos produtores rurais, profissionais da assistência técnica e outras pessoas com vínculo profissional ao meio agropecuário, atuantes no município de São João Batista do Glória/MG, no período de junho a outubro de 2009, por meio de reuniões e entrevistas.

Enquanto o percentual de produtores no Estado com produção diária acima de 500 litros é de $6,6 \%$, no município este índice é de $20,52 \%$ (Tabela 1). O levantamento realizado pelo site MilkPoint, o Top 100 MilkPoint - Base 2012, aponta que entre os 100 maiores produtores do País, 4 deles são do município de São João Batista do Glória/MG. Estes números sinalizam um diferencial do município em relação aos dados gerais do Estado de Minas Gerais.

Tabela 1 - Distribuição do número de produtores e suas produções de leite, segundo estratos de produção de leite, em percentagem.

\begin{tabular}{lcccc}
\hline \multicolumn{1}{c}{ Produção diária } & Unidade & 2009 & 2005 & $\begin{array}{c}\text { Minas } \\
\text { Gerais }\end{array}$ \\
\hline Até 50 litros & $\%$ & 7,37 & 19,21 & 44,00 \\
\hline de 51 a 200 & $\%$ & 39,48 & 40,39 & 35,40 \\
\hline de 201 a 500 & $\%$ & 32,63 & 26,49 & 14,00 \\
\hline de 501 a 1000 & $\%$ & 10,00 & 6,62 & 4,00 \\
\hline Acima de 1000 & $\%$ & 10,52 & 7,29 & 2,60 \\
\hline Total Produtores & $\%$ & 100,00 & 100,00 & 100,00 \\
\hline
\end{tabular}

Fonte: Pesquisa de Campo, Diagnóstico Municipal/Prefeitura Municipal (2005); Diagnóstico da Pecuária Leiteira em Minas Gerais, Gomes (2006) 
A partir dos fatos relevantes identificados por Canziani (2001), procurou-se quantificar a concordância ou discordância dos resultados obtidos nas reuniões, diante de uma amostra representativa de produtores de leite e profissionais da assistência técnica privada, atuantes nos sistemas de produção de leite do município. A amostra estudada foi de 21 técnicos de um universo de 23 profissionais que atuam no município, e 59 produtores participaram das reuniões, de um total de 190 produtores do município.

\section{RESULTADOS E DISCUSSÃO}

\subsection{Caracterização da Assistência Técnica}

Foram identificados 23 técnicos que prestam serviço a produtores de leite no município. Deste total, 21 participaram da pesquisa e são residentes nos municípios de Alpinópolis/MG (1), Machado/MG (1), Passos/MG (12 técnicos), São Gonçalo Sapucaí/MG (1), São João Batista Glória/MG (3 técnicos), São João da Boa Vista/SP (1), São José do Rio Preto/SP (1) e São Sebastião do Paraíso/MG (1).

A maioria dos técnicos entrevistados possuem idade de até 30 anos (11 técnicos) e a experiência média é de 12,71 anos de formado. Entre 31 a 40 anos, 4 técnicos, entre 41 a 50 anos, 4 técnicos e apenas 2, possuem mais que 50 anos de idade. Os Médicos Veterinários são predominantes $(38,10 \%)$, seguidos pelos Zootecnistas (23,81\%) e Engenheiros Agrônomos (14,29\%) (Tabela 2). Dos entrevistados, 8 ou $38,01 \%$ possuem pós graduação na área de Bovinocultura de Leite, Nutrição ou Gestão do Agronegócio. 
Tabela 2 - Perfil dos entrevistados da Assistência Técnica quanto à formação

\begin{tabular}{lcc}
\hline Formação & $\mathrm{N}^{\circ}$ & $\%$ \\
\hline Medicina Veterinária & 8 & 38,10 \\
\hline Zootecnia & 5 & 23,81 \\
\hline Engenharia Agronômica & 3 & 14,29 \\
\hline Técnico Agrícola & 2 & 9,52 \\
\hline Outros & 3 & 14,29 \\
\hline Total & 21 & 100,00 \\
\hline
\end{tabular}

Fonte: Pesquisa de Campo

Dos entrevistados, $52,38 \%$ possuem algum tipo de vínculo comercial com empresas fornecedoras de insumos. Quanto ao vínculo de trabalho, apenas $38,10 \%$ possuem carteira assinada, $23,81 \%$ possuem contrato de trabalho formalizado, e $28,57 \%$ são autônomos (Tabela 3).

Tabela 3 - Perfil dos entrevistados da Assistência Técnica quanto ao vínculo de trabalho

\begin{tabular}{lc}
\hline \multicolumn{1}{c}{ Vinculo trabalho } & $\%$ \\
\hline Autônomo, sem convênios & 9,52 \\
\hline Autônomo, com convênios & 19,05 \\
\hline Contratado como Pessoa Jurídica & 4,76 \\
\hline Contratado como Pessoa Física & 19,05 \\
\hline Contrato com carteira de trabalho assinada & 38,10 \\
\hline Estágio com contrato formal & 9,52 \\
\hline
\end{tabular}

Fonte: Pesquisa de Campo

Os técnicos que recebem menos de um salário mínimo $(9,52 \%)$ são estagiários do curso de Engenharia Agronômica da Fundação de Ensino Superior de Passos/FESP, a qual mantém um convênio com a Cooperativa Agropecuária do Sudoeste Mineiro Ltda/Casmil, que oferece bolsas para os alunos que prestam orientação a pequenos produtores associados a ela (Tabela 4). $\mathrm{O}$ maior percentual dos entrevistados (71,43\%), recebem acima de 6 salários mínimo. A qualificação pode ser associada a remuneração, pois $42,86 \%$ dos entrevistados possuem remuneração superior a 9 
salários mínimo, e destes, 66,66\% possuem cursos de pós Graduação.

Tabela 4 - Perfil dos entrevistados da Assistência Técnica quanto a Renda Bruta

\begin{tabular}{lc}
\hline \multicolumn{1}{c}{ Renda Bruta } & $\%$ \\
\hline Recebem menos de 3 Salários Mínimo & 9,52 \\
\hline Recebem de 3 a 6 Salários Mínimo & 19,05 \\
\hline Recebem de 6 a 9 Salários Mínimo & 28,57 \\
\hline Recebem mais que 9 Salários Mínimo & 42,86 \\
\hline
\end{tabular}

Fonte: Pesquisa de Campo

Dos produtores atendidos pelos Técnicos entrevistados, que incluem produtores de leite de outros municípios, inclusive de outros estados, $38,25 \%$, respondem por $54,40 \%$ do volume de leite produzido, sendo que 15 produtores (2,73\%), com média diária de 6 mil litros de leite, respondem por $11,66 \%$ do volume de leite das propriedades atendidas por estes técnicos (Tabela 5). Em contrapartida, os $61,75 \%$ dos produtores, respondem por $45,60 \%$ da produção.

$\mathrm{Na}$ Tabela 5, nota-se que o maior percentual dos produtores, com menores produções, recebem assistência apenas quando solicitado. $\mathrm{O}$ atendimento sistemático, com programação de visitas mensais ou semanais, foi observado nos técnicos com e sem vínculo comercial, sinalizando uma tendência de atendimento pós venda, vinculado a resultados de assistência técnica que poderiam ser objeto de estudo para uma melhor avaliação. 
Tabela 5 - Frequência de realização de visitas pelos técnicos entrevistados, volume de leite produzido, média diária de produção por produtor e número de vacas atendidas.

\begin{tabular}{|c|c|c|c|c|c|c|c|}
\hline $\begin{array}{l}\text { Frequência da } \\
\text { visita }\end{array}$ & $\begin{array}{r}\mathrm{N}^{\circ} \\
\text { Prod }\end{array}$ & $\%$ & $\begin{array}{c}\text { Vol. leite } \\
\text { dia }\end{array}$ & $\%$ & $\begin{array}{l}\text { Média } \\
\text { diária }\end{array}$ & $\begin{array}{c}\text { № } \\
\text { vacas }\end{array}$ & $\%$ \\
\hline $\begin{array}{l}\text { Uma ou mais } \\
\text { vezes/semana }\end{array}$ & 15 & 2,7 & 90.000 & $\begin{array}{c}11 \\
6\end{array}$ & 6.000 & 4.800 & $\begin{array}{c}12 \\
6\end{array}$ \\
\hline Uma vez ao mês & 195 & $\begin{array}{c}35 \\
5\end{array}$ & 329.962 & $\begin{array}{c}42, \\
7\end{array}$ & 1.692 & 18.107 & $\begin{array}{c}47 \\
7\end{array}$ \\
\hline Menos de uma vez/mês & 84 & $\begin{array}{c}15 \\
3\end{array}$ & 120.000 & $\begin{array}{c}15 \\
5\end{array}$ & 1.429 & 8.300 & $\begin{array}{c}21 \\
9\end{array}$ \\
\hline Quando solicitado & 255 & $\begin{array}{c}46 \\
4\end{array}$ & 232.000 & $\begin{array}{c}30, \\
0\end{array}$ & 910 & 6.700 & $\begin{array}{c}17 \\
6\end{array}$ \\
\hline Totais & 549 & 100 & 771.962 & 100 & 1.406 & 37.907 & 100 \\
\hline
\end{tabular}

Fonte: Pesquisa de campo

\section{Atividade Grupal: Opiniões dos Produtores}

Foram realizadas quatro reuniões com produtores rurais, com grupos distintos formados conforme bairro rural nos quais se localizam suas propriedades, sendo Bairro da Capetinga, Esmeril, Palmeiras e Sede do Município, totalizando uma participação de 59 produtores de leite. Nestas reuniões, foram apresentados aos produtores os seguintes questionamentos:

1) Como avaliam a assistência técnica que recebem?

2) Como vêem a relação entre assistência técnica e a gestão de suas propriedades.

A assistência técnica que recebem foi bastante criticada segundo avaliação dos produtores, os quais argumentaram que "Assistência técnica faz pela metade", "atende mais ou menos, demora para atender", "falta sabedoria (conhecimento técnico)". Por outro lado, ficou evidente a carência de assistência técnica de profissionais como o Médico Veterinário e Nutricionista, assim como o potencial de atuação para os técnicos em "acompanhamento no custo, administração geral" e "poderia fazer acompanhamento geral", e a carência de assistência técnica para pequenos produtores já que "os grandes já possuem Assistência Técnica contratada". A assistência por meio de técnicos com vinculo comercial foi apontada, assim como a desconfiança do produtor quanto às orientações 
repassadas por estes técnicos ("vendedores"). Mas esta desconfiança é superada quanto há uma relação duradoura e de confiança.

O reconhecimento da importância da assistência técnica (AT) foi apontado, quando afirmaram que "quem não tem não toca quem não tem AT não consegue manter o negócio" e que " a Assistência Técnica resolve muitos problemas e ajuda a evitá-los".

Uma das respostas esperadas e que não houve, foi quanto a resultados efetivos, sinalizando uma possível deficiência de avaliação efetiva de resultados proporcionados pela assistência técnica.

Segundo opinião dos produtores, hoje a assistência técnica não ajuda na gestão, mas consideram que seria importante esta ajuda. Um dos produtores que recebe este tipo de orientação, reconheceu, valorizou e argumentou que "em casa ajuda e melhorou $50 \%$ ". Embora alguns produtores afirmassem que o produtor não está procurando esta ajuda, eles acreditam que a assistência técnica estaria preparada e é importante para fazer a administração, o qual deveria ir à sua propriedade mensalmente e poderia ajudar inclusive ensinando como fazer as contas.

\section{Opiniões da Assistência Técnica}

Segundo os profissionais de AT, "empresas e técnicos com conhecimento e capacitados a ter uma visão geral do sistema de produção, não somente do produto/ área de atuação específicas, esses tendem hoje a acrescentar e fazer acontecer a gestão de uma propriedade".

A "informatização", "disponibilidade de sistemas e ferramentas disponíveis", "um sistema eficiente de coleta de informações e análise/arquivamento de histórico de indicadores", "uso, pelo menos, de um fluxo de caixa e balanço patrimonial correto para a gestão financeira", "acesso a informações confiáveis do mercado de leite", "aumento do nível de informação das pessoas envolvidas" foram alguns aspectos relacionados a gestão da informação apontados por todos os técnicos, inclusive com repetição do mesmo argumento.

Aspectos como "o conhecimento ou estabelecimento de metas, objetivos, visão de futuro", "saber aonde quer chegar com seu 
empreendimento" evidenciam uma visão desejada quanto aos aspectos de gestão por parte dos técnicos", mas a " capacitação do proprietário e dos seus colaboradores dentro de uma cultura de gestão adequada", "uso de linguagem simples e motivação do pessoal", complementam os fatores facilitadores da gestão.

Se a informação foi apontada como o principal aspecto facilitador da gestão na opinião dos técnicos, ela também foi a resposta mais comum entre os fatores que mais dificultam a gestão de sistemas de produção de leite. Ressalta-se que esta carência de dados refere-se a dados gerenciais, de custos, financeiros, zootécnicos e de mercado, dificultando o processo de tomada de decisão.

Segundo os técnicos, propriedades que ainda não enxergam como empresa a sua atividade, talvez ajam desta forma por desconhecimento, talvez por dificuldade e falta de apoio. A parte de organização de um sistema de gestão também exige muita dedicação e organização, que para alguns pode ser visto como dificuldade não ter uma pessoa com esta função na propriedade.

A falta de capacitação do proprietário e dos seus colaboradores dentro de uma cultura de gestão adequada, a falta conhecimento ou estabelecimento de metas, objetivos, visão de futuro, não saber onde quer chegar com seu empreendimento; aspectos culturais, educacionais e motivacionais, tanto por parte dos produtores como dos empregados e técnicos, foram outros aspectos apontados e diretamente relacionados a definição de gestão e os princípios administrativos.

A comunicação é um ponto chave que muitas vezes limita a cadeia de eventos e execuções, segundo opiniões dos entrevistados, os quais elencam ainda o "uso de linguagens complexas, baixo nível de escolaridade dos colaboradores e falta de objetivos, metas e motivação do pessoal".

\section{Destacam-se ainda:}

- A imprevisibilidade de fatores climáticos, muitas vezes incertos, ou susceptibilidade aos fatores climáticos;

- A enorme gama de fatores que interferem na eficiência do sistema (animal, manejo, pessoas, alimentação, sanidade, etc); 
- Falta de planejamento para execução, tanto atividades de curto quando a longos prazos; Incertezas de mercado dos produtos lácteos;

- Falta de união de toda classe;

Quanto à adoção das funções administrativas (Planejamento, Organização, Direção e Controle) pelas empresas assistidas pelos entrevistados:

- Não são adotadas ou são deficientes, e grande parte das fazendas não tem a noção de como realmente são importantes e que ainda falta colocá-los em prática.

- As atividades são executadas sem planejamento, posteriormente não é feito controle para medir a viabilidade (ou quando é feito é informal) nem direcionamento para a atividade.

- São poucas as empresas que têm o interesse em efetivar a coleta de dados e o controle. Em muitas, essas coletas são falhas e o próprio proprietário não tem interesse em levar a diante este trabalho pelo nível de mão-de-obra que trabalha (baixa escolaridade e desmotivação) e o custo de manter este processo que gera demanda de tempo de trabalho.

As fazendas mais "elitizadas", na opinião de um dos técnicos fazem maior uso do planejamento e organização. Se preocupam com a direção, mas possuem dificuldades quanto ao controle. Pelos argumentos, fica a dúvida se ações práticas e efetivas de planejamento, organização e direção realmente foram identificadas nas fazendas atendidas, uma vez que se trata das mesmas fazendas atendidas pelos demais técnicos.

Os técnicos foram unânimes ao afirmar que se sentem preparados para auxiliar os produtores rurais na gestão de seus sistemas de produção de leite. Entre a principal justificativa está a capacitação, mas a maioria não cita capacitação na área de gestão embora um dos entrevistados mencione a participação em um curso de gestão empresarial e que "só com a formação em cursos de ciências agrárias eu não estaria apto a auxiliar os produtores rurais na gestão de seus sistemas de produção de leite". A experiência, vivência dos problemas no dia a dia das fazendas e a capacitação 
"na área", foram os argumentos utilizados para justificar a preparação dos técnicos.

\section{CONCLUSÃO}

A maioria dos entrevistados (52,38\%) possui algum tipo de vínculo comercial com empresas fornecedoras de insumos, mas nota-se que o vinculo de trabalho passa por transformações.

As maiores remunerações dos técnicos estão associadas ao maior grau de instrução.

$\mathrm{Na}$ opinião dos técnicos, eles estariam aptos a auxiliar os produtores rurais na gestão de seus sistemas de produção de leite, o que não foi confirmado por meio de capacitação na área de gestão.

Os produtores rurais normalmente não permitem o acesso dos técnicos às informações relacionadas a gastos familiares, mesmo quando esse assunto representa um problema administrativo importante na empresa agropecuária. Uma restrição à maior participação dos técnicos no processo administrativo das empresas agropecuárias diz respeito ao sigilo de informações, aliado ao vínculo comercial que a maioria dos técnicos possui, e uma relativa insegurança dos produtores rurais em expressar sua opinião sobre o assunto.

A baixa participação dos técnicos no processo administrativo da empresa agropecuária se deve muito mais a falta de abertura e aceitação dos produtores rurais quanto a esta participação, do que pela falta de interesse dos técnicos sobre o problema, embora esta preparação do técnico não tenha se confirmado na pesquisa; ao contrário dos técnicos, os produtores rurais concordam que as questões administrativas da empresa agropecuária são sua responsabilidade, e que os técnicos devem priorizar as questões tecnológicas, em sua prestação de serviço às propriedades rurais;

O baixo uso de técnicas de gestão nos sistemas de produção leiteira possui uma relação com o baixo envolvimento dos profissionais da assistência técnica com as questões administrativas dessas empresas que exploram estes sistemas, embora existam diferentes opiniões e percepções sobre o problema, por parte dos produtores e dos profissionais da assistência técnica. 


\section{REFERÊNCIAS BIBLIOGRÁFICAS}

BATALHA, M. O.; MARCHESINI, M. M. P.; RINALDI, R. N.; MOURA, T. L.. O agronegócio brasileiro e a relação entre o perfil do profissional demandado pelos segmentos agroindustriais e o ofertado pelas instituições de ensino superior. XXV Encontro Nac. de Eng. de Produção - Porto Alegre, RS, Brasil, 29 out a 01 de Nov. de 2005a. Disponível em <http://www.abepro.org.br/biblioteca/ENEGEP2005_Enegep1101_05 93.pdf>.Acesso em 16/02/2010.

BATALHA, M. O.; MARCHESINI, M.M.P.; COSTA, M.A.B.; BERGAMASCHI, M.C.M.; RINALDI, R.N.; MOURA, T.L.. Recursos Humanos no Agronegócio: a evolução do perfil profissional. Jaboticabal: Editora Novos talentos, 2005.

CALLOU, A. B. F.; PIRES, M. L. L. e S.; LEITÃO, M. R. F. A.; SANTOS, M. S. T. O estado da arte do ensino da extensão rural no Brasil. Revista Extensão Rural, Santa Maria, DEAER/PPGExR CCR - UFSM, Ano XV, n. 16, Jul./Dez. 2008.

CANZIANI, J. R. F. Assessoria Administrativa a Produtores Rurais. 2001. 236f. Tese (Doutorado em Ciências) - Universidade de São Paulo, Escola Superior de Agricultura "Luiz de Queiroz". Piracicaba, 2001.

Curso Engenharia Agronômica. Centro de Ciências Agrárias: Universidade Federal de São Carlos - CCA-UFSCAR, s/d. Disponível em: <http://www.cca.ufscar.br/indexwp.php/engenharia-agronomica>. Acesso em: 24 fev 2011.

Diagnóstico Municipal Setor Rural. Prefeitura Municipal São João Batista do Glória, 2005.

GOMES, S.T. Diagnóstico da pecuária leiteira do Estado de Minas Gerais, 2005: relatório de pesquisa. - Belo Horizonte: FAEMG, 2006. 156 p.: il; 
MAIA, V. M.; ALEXANDRE, R. S.; SILVA, R. G.. Desafios à formação do profissional em ciências agrárias. Revista Educação Agrícola Superior - v.21. 21, n.1, 2006.

REICHERT, L. J. A administração rural em propriedades familiares. Teoria Evidencia Econômica, Passo Fundo, v. 5, n. 10, p. 67-86, maio 1998.

RINALDI, N.R.; BATALHA, M.O.; MOURA, T.L. Pós-Graduação em Agronegócios no Brasil: situação atual e perspectivas. RBPG, Brasília, v.4, n7, p.141-158, julho de 2007.

ROS, C. A. Os desafios para a formação dos profissionais das ciências agrárias no século XXI. Rural Semanal - Informativo da Universidade Federal Rural do Rio de Janeiro, ANO XV - 2008.

ZYLBERSTAJN, D.; SCARE, R. F. Gestão da Qualidade no Agribusiness. 1a. Edição. São Paulo: Atlas; 2003. 